\title{
A MODIFIED QUASI-BOUNDARY VALUE METHOD FOR A CLASS OF ABSTRACT PARABOLIC ILL-POSED PROBLEMS
}

\author{
M. DENCHE AND S. DJEZZAR
}

Received 14 October 2004; Accepted 9 August 2005

We study a final value problem for first-order abstract differential equation with positive self-adjoint unbounded operator coefficient. This problem is ill-posed. Perturbing the final condition, we obtain an approximate nonlocal problem depending on a small parameter. We show that the approximate problems are well posed and that their solutions converge if and only if the original problem has a classical solution. We also obtain estimates of the solutions of the approximate problems and a convergence result of these solutions. Finally, we give explicit convergence rates.

Copyright (C) 2006 M. Denche and S. Djezzar. This is an open access article distributed under the Creative Commons Attribution License, which permits unrestricted use, distribution, and reproduction in any medium, provided the original work is properly cited.

\section{Introduction}

We consider the following final value problem (FVP)

$$
\begin{gathered}
u^{\prime}(t)+A u(t)=0, \quad 0 \leq t<T \\
u(T)=f
\end{gathered}
$$

for some prescribed final value $f$ in a Hilbert space $\mathrm{H}$; where $A$ is a positive self-adjoint operator such that $0 \in \rho(A)$. Such problems are not well posed, that is, even if a unique solution exists on $[0, T]$ it need not depend continuously on the final value $f$. We note that this type of problems has been considered by many authors, using different approaches. Such authors as Lavrentiev [8], Lattès and Lions [7], Miller [10], Payne [11], and Showalter [12] have approximated (FVP) by perturbing the operator $A$.

In $[1,4,13]$ a similar problem is treated in a different way. By perturbing the final value condition, they approximated the problem (1.1), (1.2), with

$$
\begin{gathered}
u^{\prime}(t)+A u(t)=0, \quad 0<t<T, \\
u(T)+\alpha u(0)=f .
\end{gathered}
$$

Hindawi Publishing Corporation

Boundary Value Problems

Volume 2006, Article ID 37524, Pages 1-8

DOI 10.1155/BVP/2006/37524 
A similar approach known as the method of auxiliary boundary conditions was given in $[6,9]$. Also, we have to mention that the non standard conditions of the form (1.4) for parabolic equations have been considered in some recent papers $[2,3]$.

In this paper, we perturbe the final condition (1.2) to form an approximate nonlocal problem depending on a small parameter, with boundary condition containing a derivative of the same order than the equation, as follows:

$$
\begin{gathered}
u^{\prime}(t)+A u(t)=0, \quad 0<t<T, \\
u(T)-\alpha u^{\prime}(0)=f .
\end{gathered}
$$

Following [4], this method is called quasi-boundary value method, and the related approximate problem is called quasi-boundary value problem (QBVP). We show that the approximate problems are well posed and that their solutions $u_{\alpha}$ converge in $C^{1}([0, T], H)$ if and only if the original problem has a classical solution. We show that this method gives a better approximation than many other quasi reversibility type methods, for example, $[1,4,7]$. Finally, we obtain several other results, including some explicit convergence rates. The case where the operator $A$ has discrete spectrum has been treated in [5].

\section{The approximate problem}

Definition 2.1. A function $u:[0, T] \rightarrow H$ is called a classical solution of the (FVP) problem (resp., (QBVP) problem) if $u \in C^{1}([0, T], H), u(t) \in D(A)$ for every $t \in[0, T]$ and satisfies (1.1) and the final condition (1.2) (resp., the boundary condition (1.6)).

Now, let $\left\{E_{\lambda}\right\}_{\lambda>0}$ be a spectral measure associated to the operator $A$ in the Hilbert space $H$, then for all $f \in H$, we can write

$$
f=\int_{0}^{\infty} d E_{\lambda} f
$$

If the (FVP) problem (resp., (QBVP) problem) admits a solution $u$ (resp., $u_{\alpha}$ ), then this solution can be represented by

$$
u(t)=\int_{0}^{\infty} e^{\lambda(T-t)} d E_{\lambda} f
$$

respectively,

$$
u_{\alpha}(t)=\int_{0}^{\infty} \frac{e^{-\lambda t}}{\alpha \lambda+e^{-\lambda T}} d E_{\lambda} f
$$

THeORem 2.2. For all $f \in H$, the functions $u_{\alpha}$ given by (2.3) are classical solutions to the $(\mathrm{Q} B V P)$ problem and we have the following estimate

$$
\left\|u_{\alpha}(t)\right\| \leq \frac{T}{\alpha(1+\ln (T / \alpha))}\|f\|, \quad \forall t \in[0, T]
$$

where $\alpha<e T$. 
Proof. If we assume that the functions $u_{\alpha}$ given in (2.3) are defined for all $t \in[0, T]$, then, it is easy to show that $u_{\alpha} \in C^{1}([0, T], H)$ and

$$
u_{\alpha}^{\prime}(t)=\int_{0}^{\infty} \frac{-\lambda e^{-\lambda t}}{\alpha \lambda+e^{-\lambda T}} d E_{\lambda} f
$$

From

$$
\left\|A u_{\alpha}(t)\right\|^{2}=\int_{0}^{\infty} \frac{\lambda^{2} e^{-2 \lambda t}}{\left(\alpha \lambda+e^{-\lambda T}\right)^{2}} d\left\|E_{\lambda} f\right\|^{2} \leq \frac{1}{\alpha^{2}} \int_{0}^{\infty} d\left\|E_{\lambda} f\right\|^{2}=\frac{1}{\alpha^{2}}\|f\|^{2},
$$

we get $u_{\alpha}(t) \in D(A)$ and so $u_{\alpha} \in C([0, T], D(A))$. This shows that the function $u_{\alpha}$ is a classical solution to the (QBVP) problem.

Now, using (2.3), we have

$$
\left\|u_{\alpha}(t)\right\|^{2} \leq \int_{0}^{\infty} \frac{1}{\left(\alpha \lambda+e^{-\lambda T}\right)^{2}} d\left\|E_{\lambda} f\right\|^{2}
$$

if we put

$$
h(\lambda)=\left(\alpha \lambda+e^{-\lambda T}\right)^{-1}, \quad \text { for } \lambda>0,
$$

then,

$$
\sup _{\lambda>0} h(\lambda)=h\left(\frac{\ln (T / \alpha)}{T}\right)
$$

and this yields

$$
\left\|u_{\alpha}(t)\right\|^{2} \leq\left[\frac{T}{\alpha(1+\ln (T / \alpha))}\right]^{2} \int_{0}^{\infty} d\left\|E_{\lambda} f\right\|^{2}=\left[\frac{T}{\alpha(1+\ln (T / \alpha))}\right]^{2}\|f\|^{2} .
$$

This shows that the integral defining $u_{\alpha}(t)$ exists for all $t \in[0, T]$ and we have the desired estimate.

Remark 2.3. One advantage of this method of regularization is that the order of the error, introduced by small changes in the final value $f$, is less than the order given in [4].

Now, we give the following convergence result.

Theorem 2.4. For every $f \in H, u_{\alpha}(T)$ converges to $f$ in $H$, as $\alpha$ tends to zero.

Proof. Let $\varepsilon>0$, choose $\eta>0$ for which

$$
\int_{\eta}^{\infty} d\left\|E_{\lambda} f\right\|^{2}<\frac{\varepsilon}{2}
$$

From (2.3), we have

$$
\left\|u_{\alpha}(T)-f\right\|^{2} \leq \alpha^{2} \int_{0}^{\eta} \frac{\lambda^{2}}{\left(\alpha \lambda+e^{-\lambda T}\right)^{2}} d\left\|E_{\lambda} f\right\|^{2}+\frac{\varepsilon}{2},
$$


4 Regularization of parabolic ill-posed problems

so by choosing $\alpha$ such that

$$
\alpha^{2}<\varepsilon\left(2 \int_{0}^{\eta} \lambda^{2} e^{2 \lambda T}\left\|E_{\lambda} f\right\|^{2}\right)^{-1}
$$

we obtain the desired result.

Theorem 2.5. For every $f \in H$, the (FVP) problem has a classical solution u given by (2.2), if and only if the sequence $\left(u_{\alpha}^{\prime}(0)\right)_{\alpha>0}$ converge in $H$. Furthermore, we then have that $u_{\alpha}(t)$ converges to $u(t)$ in $C^{1}([0, T], H)$ as $\alpha$ tends to zero.

Proof. If we assume that the (FVP) problem has a classical solution $u$, then we have

$$
\begin{aligned}
\left\|u_{\alpha}^{\prime}(0)-u^{\prime}(0)\right\|^{2} & =\int_{0}^{\infty} \frac{\alpha^{2} \lambda^{4} e^{2 \lambda T}}{\left(\alpha \lambda+e^{-\lambda T}\right)^{2}}\left\|d E_{\lambda} f\right\|^{2} \\
& \leq \alpha^{2} \int_{0}^{\eta} \lambda^{4} e^{4 \lambda T} d\left\|E_{\lambda} f\right\|^{2}+\int_{\eta}^{\infty} \frac{\alpha^{2} \lambda^{4} e^{2 \lambda T}}{\alpha^{2} \lambda^{2}} d\left\|E_{\lambda} f\right\|^{2} \\
& <\alpha^{2} \int_{0}^{\eta} \lambda^{4} e^{4 \lambda T} d\left\|E_{\lambda} f\right\|^{2}+\frac{\varepsilon}{2}
\end{aligned}
$$

so by choosing $\alpha$ such that $\alpha^{2}<\varepsilon\left(2 \int_{0}^{\eta} \lambda^{4} e^{4 \lambda T} d\left\|E_{\lambda} f\right\|^{2}\right)^{-1}$, we obtain

$$
\left\|u_{\alpha}^{\prime}(0)-u^{\prime}(0)\right\|^{2}<\varepsilon,
$$

this shows that $\left\|u_{\alpha}^{\prime}(0)-u^{\prime}(0)\right\|$ tends to zero as $\alpha$ tends to zero. Since

$$
\begin{aligned}
\left\|u_{\alpha}^{\prime}(t)-u^{\prime}(t)\right\|^{2} & \leq \int_{0}^{\infty} \lambda^{2}\left(\frac{1}{\alpha \lambda+e^{-\lambda T}}-e^{\lambda T}\right)^{2} d\left\|E_{\lambda} f\right\|^{2} \\
& =\left\|u_{\alpha}^{\prime}(0)-u^{\prime}(0)\right\|^{2}
\end{aligned}
$$

then $u_{\alpha}^{\prime}(t)$ converges to $u^{\prime}(t)$ uniformly in $[0, T]$ as $\alpha$ tends to zero.

Since

$$
\left\|u_{\alpha}(0)-u(0)\right\|^{2} \leq \alpha^{2} \int_{0}^{\eta} \lambda^{2} e^{4 \lambda T} d\left\|E_{\lambda} f\right\|^{2}+\frac{\varepsilon}{2},
$$

for $\eta$ quite large. Then by choosing $\alpha$ such that $\alpha^{2}<\left(2 \int_{0}^{\eta} \lambda^{2} e^{4 \lambda T} d\left\|E_{\lambda} f\right\|^{2}\right)^{-1}$, we get

$$
\left\|u_{\alpha}(0)-u(0)\right\|^{2}<\varepsilon
$$

Thus $u_{\alpha}(0)$ converges to $u(0)$, which in turn gives that $u_{\alpha}(t)$ converges to $u(t)$ uniformly in $[0, T]$ as $\alpha$ tends to zero. Combining all these convergence results, we conclude that $u_{\alpha}(t)$ converges to $u(t)$ in $C^{1}([0, T], H)$.

Now, assume that $\left(u_{\alpha}^{\prime}(0)\right)_{\alpha>0}$ converges in $H$. Since $u_{\alpha}$ is a classical solution to the (QBVP) problem, then we have

$$
\left\|u_{\alpha}^{\prime}(0)\right\|^{2}=\int_{0}^{\infty} \frac{\lambda^{2}}{\left(\alpha \lambda+e^{-\lambda T}\right)^{2}} d\left\|E_{\lambda} f\right\|^{2},
$$


and it is easy to show that

$$
\left\|\lim _{\alpha \downarrow 0} u_{\alpha}^{\prime}(0)\right\|^{2}=\int_{0}^{\infty} \lambda^{2} e^{2 \lambda T} d\left\|E_{\lambda} f\right\|^{2},
$$

and so the function $u(t)$ defined by

$$
u(t)=\int_{0}^{\infty} e^{\lambda(T-t)} d E_{\lambda} f
$$

is a classical solution to the (FVP) problem. This ends the proof of the theorem.

THeOREm 2.6. If the function $u$ given by (2.2) is a classical solution of the (FVP) problem, and $u_{\alpha}^{\delta}$ is a solution of the $(Q B V P)$ problem for $f=f_{\delta}$, such that $\left\|f-f_{\delta}\right\|<\delta$, then we have

$$
\left\|u(0)-u_{\alpha}^{\delta}(0)\right\| \leq c\left(1+\ln \frac{T}{\delta}\right)^{-1}
$$

where $c=T(1+\|A u(0)\|)$.

Proof. Suppose that the function $u$ given by (2.2) is a classical solution to the (FVP) problem, and let's denote by $u_{\alpha}^{\delta}$ a solution of the (QBVP) problem for $f=f_{\delta}$, such that

$$
\left\|f-f_{\delta}\right\|<\delta
$$

Then, $u_{\alpha}^{\delta}(t)$ is given by

$$
u_{\alpha}^{\delta}(t)=\int_{0}^{\infty} \frac{e^{-\lambda t}}{\alpha \lambda+e^{-\lambda T}} d E_{\lambda} f_{\delta}, \quad \forall t \in[0, T] .
$$

From (2.2) and (2.24), we have

$$
\left\|u(0)-u_{\alpha}^{\delta}(0)\right\| \leq \Delta_{1}+\Delta_{2},
$$

where $\Delta_{1}=\left\|u(0)-u_{\alpha}(0)\right\|$, and $\Delta_{2}=\left\|u_{\alpha}(0)-u_{\alpha}^{\delta}(0)\right\|$. Using (2.9), we get

$$
\begin{aligned}
& \Delta_{1} \leq \frac{T}{(1+\ln (T / \alpha))}\left(\int_{0}^{\infty} \lambda^{2} e^{2 \lambda T} d\left\|E_{\lambda} f\right\|^{2}\right)^{1 / 2}, \\
& \Delta_{2} \leq \frac{T}{\alpha(1+\ln (T / \alpha))}\left\|f-f_{\delta}\right\|,
\end{aligned}
$$

then,

$$
\begin{aligned}
\Delta_{1} & \leq \frac{T\|A u(0)\|}{1+\ln (T / \alpha)} \\
\Delta_{2} & \leq \frac{T \delta}{\alpha(1+\ln (T / \alpha))}
\end{aligned}
$$

From (2.27), we obtain

$$
\left\|u_{\alpha}(0)-u_{\alpha}^{\delta}(0)\right\|^{2} \leq \frac{T\|A u(0)\|}{(1+\ln (T / \alpha))}+\frac{T \delta}{\alpha(1+\ln (T / \alpha))},
$$


6 Regularization of parabolic ill-posed problems

then, for the choice $\alpha=\delta$, we get

$$
\left\|u_{\alpha}(0)-u_{\alpha}^{\delta}(0)\right\|^{2} \leq \frac{T(1+\|A u(0)\|)}{(1+\ln (T / \alpha))} .
$$

Remark 2.7. From (2.22), for $T>e^{-1}$ we get

$$
\left\|u(0)-u_{\alpha}^{\delta}(0)\right\| \leq c\left(\ln \frac{1}{\delta}\right)^{-1}
$$

Remark 2.8. Under the hypothesis of the above theorem, if we denote by $U_{\alpha}^{\delta}$ the solution of the approximate (FVP) problem for $f=f_{\delta}$, using the quasireversibility method [7], we obtain the following estimate

$$
\left\|u(0)-U_{\alpha}^{\delta}(0)\right\| \leq c_{1}\left(\ln \frac{1}{\delta}\right)^{-2 / 3} .
$$

Proof. A proof can be given in a similar way as in [9].

THeOREm 2.9. If there exists an $\varepsilon \in] 0,2[$ so that

$$
\int_{0}^{\infty} \lambda^{\varepsilon} e^{\varepsilon \lambda T}\left\|d E_{\lambda} f\right\|^{2}
$$

converges, then $u_{\alpha}(T)$ converges to $f$ with order $\alpha^{\varepsilon} \mathcal{E}^{-2}$ as $\alpha$ tends to zero.

Proof. Let $\varepsilon \in] 0,2\left[\right.$ such that $\int_{0}^{\infty} \lambda^{\varepsilon} e^{\varepsilon \lambda T}\left\|d E_{\lambda} f\right\|^{2}$ converges, and let $\left.\beta \in\right] 0,2[$. For a fix $\lambda>0$, and if we define a function $g_{\lambda}(\alpha)=\alpha^{\beta} /\left(\alpha \lambda+e^{-\lambda T}\right)^{2}$. Then we can show that

$$
g_{\lambda}(\alpha) \leq g_{\lambda}\left(\alpha_{0}\right), \quad \forall \alpha>0,
$$

where $\alpha_{0}=\beta e^{-\lambda T} /(2-\beta) \lambda$. Furthermore, from (2.3), we have

$$
\left\|u_{\alpha}(T)-f\right\|^{2}=\alpha^{2-\beta} \int_{0}^{\infty} \lambda^{2} g_{\lambda}(\alpha) d E_{\lambda} f .
$$

Hence from (2.33) and (2.34) we obtain

$$
\left\|u_{\alpha}(T)-f\right\|^{2} \leq \alpha^{2-\beta}\left(\frac{\beta}{2-\beta}\right)^{\beta} \int_{0}^{\infty} \lambda^{2-\beta} e^{(2-\beta) \lambda T} d\left\|E_{\lambda} f\right\|^{2} .
$$

If we choose $\beta=(2-\varepsilon)$, we have

$$
\left\|u_{\alpha}(T)-f\right\|^{2} \leq \alpha^{\varepsilon} \varepsilon^{-2}\left(4 \int_{0}^{\infty} \lambda^{\varepsilon} e^{\varepsilon \lambda T} d\left\|E_{\lambda} f\right\|^{2}\right),
$$

hence

$$
\left\|u_{\alpha}(T)-f\right\|^{2} \leq c_{\varepsilon} \alpha^{\varepsilon} \varepsilon^{-2}
$$

with $c_{\varepsilon}=4 \int_{0}^{\infty} \lambda^{\varepsilon} e^{\varepsilon \lambda T} d\left\|E_{\lambda} f\right\|^{2}$. 
Now, we give the following corollary.

Corollary 2.10. If there exists an $\varepsilon \in] 0,2[$ so that

$$
\int_{0}^{\infty} \lambda^{(\varepsilon+2 \gamma)} e^{(\varepsilon+2) \lambda T} d\left\|E_{\lambda} f\right\|^{2}
$$

where $\gamma=\overline{0,1}$, converges, then $u_{\alpha}$ converges to $u$ in $C^{1}([0, T], H)$ with order of convergence $\alpha^{\varepsilon} \mathcal{E}^{-2}$.

Proof. If we assume that (2.38) is satisfied, then

$$
\int_{0}^{\infty} \lambda^{2} e^{2 \lambda T} d\left\|E_{\lambda} f\right\|^{2}
$$

converges, and so the function $u(t)$ given by (2.2) is a classical solution of the (FVP) problem. Let $u_{\alpha}^{(\gamma)}, u^{(\gamma)}$ denote the derivatives of order $\gamma(\gamma=\overline{0,1})$ of the functions $u_{\alpha}$ and $u$, respectively. Using the following inequalities

$$
\begin{aligned}
\left\|u_{\alpha}^{(\gamma)}(0)-u^{(\gamma)}(0)\right\|^{2} & =\int_{0}^{\infty} \frac{\alpha^{2} \lambda^{(2+2 \gamma)} e^{2 \lambda T}}{\left(\alpha \lambda+e^{-\lambda T}\right)^{2}} d\left\|E_{\lambda} f\right\|^{2} \\
& \leq \alpha^{2-\beta}\left(\frac{\beta}{2-\beta}\right)^{\beta} \int_{0}^{\infty} \lambda^{(2+2 \gamma-\beta)} e^{(4-\beta) \lambda T} d\left\|E_{\lambda} f\right\|^{2},
\end{aligned}
$$

and setting $\beta=2-\varepsilon$, in (2.40), we obtain

$$
\left\|u_{\alpha}^{(\gamma)}(0)-u^{(\gamma)}(0)\right\|^{2} \leq c_{\varepsilon, \gamma} \alpha^{\varepsilon} \varepsilon^{-2},
$$

where $c_{\varepsilon, \gamma}=4 \int_{0}^{\infty} \lambda^{(\varepsilon+2 \gamma)} e^{(\varepsilon+2) \lambda T} d\left\|E_{\lambda} f\right\|^{2}$.

And since

$$
\left\|u_{\alpha}^{(\gamma)}(t)-u^{(\gamma)}(t)\right\|^{2} \leq\left\|u_{\alpha}^{(\gamma)}(0)-u^{(\gamma)}(0)\right\|^{2}
$$

then $u_{\alpha}^{(\gamma)}(t)$ converges to $u^{(\gamma)}(t)$ uniformly in $[0, T]$, with order of convergence $\alpha^{\varepsilon} \varepsilon^{-2}$, and so $u_{\alpha}$ converges to $u$ in $C^{1}([0, T], H)$, with order $\alpha^{\varepsilon} \mathcal{E}^{-2}$.

\section{References}

[1] M. Ababna, Regularization by nonlocal conditions of the problem of the control of the initial condition for evolution operator-differential equations, Vestnik Belorusskogo Gosudarstvennogo Universiteta. Seriya 1. Fizika, Matematika, Informatika (1998), no. 2, 60-63, 81 (Russian).

[2] K. A. Ames and L. E. Payne, Asymptotic behavior for two regularizations of the Cauchy problem for the backward heat equation, Mathematical Models \& Methods in Applied Sciences 8 (1998), no. $1,187-202$.

[3] K. A. Ames, L. E. Payne, and P. W. Schaefer, Energy and pointwise bounds in some non-standard parabolic problems, Proceedings of the Royal Society of Edinburgh. Section A. Mathematics 134 (2004), no. 1, 1-9.

[4] G. W. Clark and S. F. Oppenheimer, Quasireversibility methods for non-well-posed problems, Electronic Journal of Differential Equations 1994 (1994), no. 8, 1-9. 
[5] M. Denche and K. Bessila, A modified quasi-boundary value method for ill-posed problems, Journal of Mathematical Analysis and Applications 301 (2005), no. 2, 419-426.

[6] V. K. Ivanov, I. V. Mel'nikova, and A. I. Filinkov, Operator-Differential Equations and Ill-Posed Problems, Fizmatlit "Nauka", Moscow, 1995.

[7] R. Lattès and J.-L. Lions, Méthode de Quasi-Réversibilité et Applications, Travaux et Recherches Mathématiques, no. 15, Dunod, Paris, 1967.

[8] M. M. Lavrentiev, Some Improperly Posed Problems of Mathematical Physics, Springer Tracts in Natural Philosophy, vol. 11, Springer, Berlin, 1967.

[9] I. V. Mel'nikova, Regularization of ill-posed differential problems, Sibirskiŭ Matematicheskiĭ Zhurnal 33 (1992), no. 2, 125-134, 221 (Russian), translated in Siberian Math. J. 33 (1992), no. 2, 289-298.

[10] K. Miller, Stabilized quasi-reversibility and other nearly-best-possible methods for non-well-posed problems, Symposium on Non-Well-Posed Problems and Logarithmic Convexity (Heriot-Watt Univ., Edinburgh, 1972), Lecture Notes in Mathematics, vol. 316, Springer, Berlin, 1973, pp. 161-176.

[11] L. E. Payne, Some general remarks on improperly posed problems for partial differential equations, Symposium on Non-Well-Posed Problems and Logarithmic Convexity (Heriot-Watt Univ., Edinburgh, 1972), Lecture Notes in Mathematics, vol. 316, Springer, Berlin, 1973, pp. 1-30.

[12] R. E. Showalter, The final value problem for evolution equations, Journal of Mathematical Analysis and Applications 47 (1974), no. 3, 563-572.

[13] _ Cauchy problem for hyperparabolic partial differential equations, Trends in the Theory and Practice of Nonlinear Analysis (Arlington, Tex, 1984), North-Holland Math. Stud., vol. 110, North-Holland, Amsterdam, 1985, pp. 421-425.

M. Denche: Laboratoire Equations Differentielles, Département de Mathématiques, Faculté des Sciences, Université Mentouri Constantine, 25000 Constantine, Algeria E-mail address: denech@wissal.dz

S. Djezzar: Laboratoire Equations Differentielles, Département de Mathématiques, Faculté des Sciences, Université Mentouri Constantine, 25000 Constantine, Algeria E-mail address: salah_djezzar@yahoo.fr 